\title{
LA NECESIDAD DE MEDIR LA CULTURA ORGANIZACIONAL. UNA REVISIÓN DEL TEMA
}

\section{THE NEED TO MEASURE ORGANIZATIONAL CULTURE.A REVIEW OF THE TOPIC}

\author{
Msc José Gregorio Arévalo Ascanio ${ }^{\mathrm{a}}$, Msc Genny Torcoroma Navarro Claro ${ }^{\mathrm{b}}$, Msc Ramón \\ Armando Bayona Trillos ${ }^{\mathrm{c}}$
}

\begin{abstract}
a Universidad Francisco de Paula Santander Seccional Ocaña. Grupo de Investigación en Desarrollo Socioempresarial (GIDSE). Docente de tiempo completo . Ocaña, Norte de Santander, Colombia, Tel.:+(57-7)- 5690088. jgarevaloa@ufpso.edu.co

${ }^{b}$ Universidad Francisco de Paula Santander Seccional Ocaña . Grupo de Investigación en Desarrollo Socioempresarial (GIDSE). Docente Ocasional. Ocaña, Norte de Santander, Colombia,Tel.:+(57-7)-5690088.gtnavarroc@ufpso.edu.co

${ }^{c}$ Universidad Francisco de Paula Santander Seccional Ocaña. Grupo de Investigación en Desarrollo Socioempresarial (GIDSE). Docente de tiempo completo. Ocaña, Norte de Santander, Colombia,Tel.:+(57-7)-5690088.rabayonat@ufpso.edu.co
\end{abstract}

\begin{abstract}
Resumen: En la actualidad, sociólogos, psicólogos, académicos y/o empresarios se han preocupado por estudiar la cultura de las organizaciones con el propósito de mejorar su desempeño, ya que dicho constructo está estrechamente ligado con el éxito empresarial. Sin embargo, aún son muchas las organizaciones que todavía no han mostrado su interés en estudiarla y mucho menos en medirla. El objetivo de este artículo es presentar los resultados de la revisión y el análisis del tema de la cultura organizacional y los modelos y métodos para su respectiva medición. El documento se divide en tres partes. En la primera se hace una aproximación al concepto de cultura organizacional. En la segunda, se revisa los documentos empíricos y teóricos hallados sobre el evento de estudio en las bases de datos Science Direct, Ebsco, Scielo y Redalyc, junto con el proceso metodológico estructurado de revisión bibliográfica. En la tercera parte, se presenta una síntesis de los principales modelos de medición de la cultura organizacional, en el que se hace una mención especial al modelo de valores en competencia de Kim Cameron y Robert Quinn. Para finalizar, se muestra unas conclusiones en las que se resalta la utilidad del artículo, por incorporar un referente teórico y empírico sobre la cultura organizacional y sus modelos de medición en el contexto empresarial.
\end{abstract}


Palabras clave: Cultura organizacional, modelos de medición, métodos de medición, modelo de valores en competencia.

\begin{abstract}
Currently, sociologists, psychologists, academics and / or entrepreneurs have been concerned with studying the culture of organizations for the purpose of improving their performance, since this construct is closely linked to business success. However, there are still many organizations that have not yet shown an interest in studying it, much less in measuring it. The objective of this article is to present the results of the review and analysis of the subject of the organizational culture and the models and methods for their respective measurement. The document is divided into three parts. In the first, an approach to the concept of organizational culture is made. In the second, we review the empirical and theoretical documents found on the study event in the databases Science Direct, Ebsco, Scielo, and Redalyc, along with the structured methodological method of bibliographic review. In the third part, a synthesis of the main models of measurement of the organizational culture is presented, in which special mention is given to the competing values model of Kim Cameron and Robert Quinn. Finally, we show some conclusions that highlight the utility of the article, to incorporate a theoretical and empirical reference on the organizational culture and its measurement models in the business context.
\end{abstract}

Keywords: Organizational culture, measurement models, measurement methods, Competing Values Framework.

\section{INTRODUCCIÓN}

Este trabajo pretende ilustrar el tema de la cultura organizacional junto con los modelos y métodos de medición de la misma, a partir de una revisión bibliográfica en libros de texto $\mathrm{y}$ en revistas de alto impacto publicados en las bases de datos Science Direct, Ebsco, Scielo y Redalyc. La estructura y el contenido de este artículo dejan ver la existencia de estudios tanto de índole teóricos como empíricos en la literatura científica. La variable de cultura organizacional es uno de los temas de mayor interés a ser estudiado por parte de profesionales de distintas disciplinas como la psicología, la sociología y la administración, prueba de ello se halla en la afirmación de (Hernández \& Méndez, 2012), quienes indican que la cultura organizacional se ha convertido en uno de los conceptos centrales dentro de las teorías organizacionales en los últimos 25 años. La revisión realizada sigue una metodología en la que se busca identificar las orientaciones teóricas respecto a la cultura organizacional y sus modelos de medición, se sintetiza la literatura representativa sobre los eventos de estudio, en lo cual se generó como resultado una breve evidencia de las diferentes formas de conceptualizar la cultura, encontrándose que en las definiciones incorporadas se pueden identificar con claridad tres elementos usados para explicarla: creencias, valores, costumbres y rituales. Seguidamente, se presenta el resultado de la revisión realizada. Se muestra el análisis de las investigaciones que sobre el tema se han realizado por parte de la comunidad científica, en donde se reportan 1.497 documentos usando los descriptores "cultura organizacional" y "modelos de medición de cultura" (desde inicios del año 2005 hasta abril de 2017). Es preciso aclarar que para 
efectos de este documento de seleccionaron (5 empíricos y 5 teóricos) que se relacionaban de forma directa con las categorías planteadas. En las conclusiones se deja ver que difícilmente se van encontrar un modelo, un método o un instrumento que sea capaz de medir de manera precisa y exacta la cultura organizacional de cualquier tipo de empresa.

\section{Estado del arte y fundamentación teórica. Aproximación al concepto de cultura organizacional}

Respecto a la indagación sobre el origen etimológico de la palabra cultura, hay que decir que proviene del latín cultūra y su aplicación se ha dado en el cultivo del hombre en conocimiento y formación, representando los procesos de formación de las civilizaciones (Universidad de la Experiencia de Zaragoza, 2017).

En el Diccionario de la Real Academia Española se define cultura principalmente con dos sentidos, primero como el conjunto de conocimientos que permite a alguien desarrollar su juicio crítico y segundo como el conjunto de modos de vida y costumbres, conocimientos y grado de desarrollo artístico, científico, industrial, en una época, grupo social (2001, pág. 714). Por su parte, en el diccionario de Ultimate Business de Cambridge, la cultura organizacional se define como: los tipos de actitudes y formas de trabajo acordadas entre los empleados de una empresa u organización (Cambridge University Press, 2017).

Partiendo de lo anterior y teniendo en cuenta la aclaración etimológica y las definiciones de los diccionarios consultados, se entra de lleno al objetivo de indagar sobre las definiciones de cultura organizacional dadas por los autores de mayor prestigio $\mathrm{y}$ reconocimiento en la comunidad académica. Sin antes olvidar que, aunque es probable que quien haya usado por primera vez el termino de cultura organizacional haya sido Andrew Pettigrew en 1979 y que su mayor difusión se le debe a Edgar Schein quien señaló algunos usos habituales del concepto de cultura (Rodríguez, 2001).

Vale la pena señalar, que dentro de la multitud de conceptos existentes sobre esta temática se seleccionaron algunos que siguen teniendo vigencia. Las definiciones encontradas en diferentes textos consultados (véase Tabla 1) tienen una postura homogénea sobre la definición de cultura organizacional.

Tabla 1. Definiciones de cultura organizacional

\begin{tabular}{|c|c|}
\hline Autor & Definición \\
\hline $\begin{array}{l}\text { (Schein, } \\
\text { 2009) }\end{array}$ & $\begin{array}{l}\text { "patrón de suposiciones tácitas } \\
\text { compartidas que fue aprendido } \\
\text { por un grupo, ya que resolvió } \\
\text { los problemas de adaptación } \\
\text { externa y de integración } \\
\text { interna, que ha funcionado lo } \\
\text { suficientemente bien como } \\
\text { para ser considerado válido y, } \\
\text { por lo tanto, para ser enseñado } \\
\text { a los nuevos miembros como la } \\
\text { forma correcta Para percibir, } \\
\text { pensar y sentir en relación con } \\
\text { esos problemas" }\end{array}$ \\
\hline $\begin{array}{l}\text { (Peters \& } \\
\text { Waterman, } \\
1984)\end{array}$ & $\begin{array}{l}\text { "conjunto dominante y } \\
\text { coherente de valores } \\
\text { compartidos transmitidos por } \\
\text { significados simbólicos como } \\
\text { cuentos, mitos, leyendas, } \\
\text { slogans y anécdotas". }\end{array}$ \\
\hline $\begin{array}{l}\text { (Stephen, } \\
\text { 2004) }\end{array}$ & $\begin{array}{l}\text { "define la cultura de una } \\
\text { organización como aquella } \\
\text { cultura que expresa los valores } \\
\text { centrales que comparten la }\end{array}$ \\
\hline
\end{tabular}




\begin{tabular}{|c|c|}
\hline & $\begin{array}{l}\text { mayoría de los miembros de la } \\
\text { organización". }\end{array}$ \\
\hline $\begin{array}{l}\text { Hofstede } \\
\text { citado por } \\
\text { (Ruiz \& } \\
\text { Naranjo, } \\
\text { 2012) }\end{array}$ & $\begin{array}{l}\text { "programa mental colectivo } \\
\text { que distingue a los miembros } \\
\text { de un grupo o categoría de } \\
\text { otro. Es compartida por } \\
\text { personas cuya vivencia está } \\
\text { dentro del mismo ambiente } \\
\text { social donde fue aprendida". }\end{array}$ \\
\hline $\begin{array}{l}\text { (Toca \& } \\
\text { Carrillo, } \\
\text { 2009) }\end{array}$ & $\begin{array}{l}\text { "conjunto de instituciones } \\
\text { informales compartidas que } \\
\text { gobiernan y legitiman la } \\
\text { conducta de una colectividad } \\
\text { organizacional". }\end{array}$ \\
\hline $\begin{array}{l}\text { (Góngora, } \\
\text { Nóbile, \& } \\
\text { Reija, } \\
\text { 2014) }\end{array}$ & $\begin{array}{l}\text { "el conjunto de normas, } \\
\text { creencias, valores, costumbres, } \\
\text { rituales, lenguajes, artefactos y } \\
\text { presunciones básicas existentes } \\
\text { en una organización". }\end{array}$ \\
\hline $\begin{array}{l}\text { (Serna, } \\
\text { 2003) }\end{array}$ & $\begin{array}{l}\text { "La cultura de una institución, } \\
\text { es la manera como las } \\
\text { organizaciones hacen las cosas, } \\
\text { como establecen prioridades y } \\
\text { dan importancia a las } \\
\text { diferentes tareas empresariales, } \\
\text { además de incluir lo que es } \\
\text { importante para la empresa." }\end{array}$ \\
\hline $\begin{array}{l}\text { (Porras, } \\
2009)\end{array}$ & $\begin{array}{l}\text { "es un sistema o conjunto } \\
\text { central de convicciones, en } \\
\text { términos de creencias y } \\
\text { valores, que se construyen } \\
\text { colectivamente a partir de la } \\
\text { experiencia diaria, es decir a } \\
\text { través de la interacción } \\
\text { simbólica, y que regulan, } \\
\text { orientan, condicionan y } \\
\text { determinan las percepciones, } \\
\text { los pensamientos, los } \\
\text { sentimientos y las acciones } \\
\text { (actuaciones) de las personas } \\
\text { miembros de un grupo social u } \\
\text { organización productiva en } \\
\text { este caso". }\end{array}$ \\
\hline $\begin{array}{l}\text { (Aktouf, } \\
\text { 2002) }\end{array}$ & $\begin{array}{l}\text { "conjunto de evidencias" o un } \\
\text { "conjunto de postulados" } \\
\text { compartidos por los miembros, }\end{array}$ \\
\hline
\end{tabular}

\begin{tabular}{|l|l|}
\hline dirigentes y empleados. Sería \\
un "cemento" que "mantiene la \\
organización como un todo", \\
que le da un "sentido" y un \\
"sentido de identidad" para sus \\
miembros.
\end{tabular}

Fuente: elaboración propia a partir de diversos autores.

En las definiciones presentadas se pueden identificar con claridad tres elementos usados para explicar la cultura organizacional:

- Creencias.

- Valores.

- Costumbres y rituales.

Sin embargo, aunque difícilmente se va encontrar un concepto igual a otro, lo cierto es que la mayoría de quienes han escrito sobre este concepto, están de acuerdo en que la cultura es algo que es compartido por los miembros de la organización, que influye en la manera en que estos se comportan y que diferencia notoriamente a una organización de otra.

Para los propósitos de este escrito se asume, a manera de síntesis, que la cultura de una organización se refiere a un intangible que debe ser interiorizado y del que hacen parte las creencias, los valores, las costumbres y rituales que reflejan la mentalidad que predomina en la organización y que a su vez le garantiza la heterogeneidad.

\subsection{Investigaciones sobre cultura organizacional}

Se realizó una revisión sistemática en bases de datos especializadas, a partir de categorías como cultura organizacional, medición de la cultura e instrumentos para medir la cultura; y se establecieron los 
siguientes límites: artículos publicados en revistas indexadas y publicaciones (empíricas y teóricas), así como tesis de universidades cuya temática fuera aplicable al contexto.

En términos metodológicos, se revisaron las orientaciones teóricas respecto a la cultura organizacional, compendiando la literatura representativa sobre la temática en un periodo dado. Lo que significa que, el presente trabajo es una investigación teórico descriptiva de tipo documental (ver figura 1), sobre el tema de cultura organizacional. Es pertinente aclarar que la revisión documental se realizó en libros de texto y revistas de alto impacto publicados en las bases de datos Science Direct, Ebsco, Scielo, y Redalyc.

\section{Identificación de} Bibliografía relevante

- Objetivo: Abarcar los conceptos de mayor importancia desarrollados respecto a cultura organizacional y sus modelos de medición.

-Actividades: Seleccionar libros de texto y revistas de mayor impacto / Analizar la bibliografía / Hacer análisis de contenido.

\section{Generación de áreas}

\section{temáticas}

-Objetivo: Identificar las relaciones mediante un análisis de contenido.

-Actividades: Realiazar fichas para cada artículo cientifico / Identificar agrupaciones temáticas.

\section{Interrelación entre los} documentos

-Objetivo: Desarrollar relaciones entre las áreas temáticas.

- Actividades: Relacionar las distintas áreas temáticas / Hacer conclusiones sobre la cultura organizacional y sus modelos de medición.
Figura 1. Proceso estructurado de revisión bibliográfica

Fuente: elaboración propia a partir de (Romero, Matamoros, \& Campo, 2013)

En la literatura se obtienen múltiples estudios de cultura organizacional, solo en las bases de datos Science Direct y Ebsco se reportan 1.497 documentos usando los descriptores "cultura organizacional" y "modelos de medición de cultura" (desde inicios del año 2005 hasta abril de 2017). Para propósito de este artículo sólo se presentan (5 empíricos y 5 teóricos) que se relacionaban de forma directa con las categorías planteadas.

Tabla 2. Documentos empíricos revisados

\begin{tabular}{|c|c|c|}
\hline No. & Autores & Título \\
\hline 1. & $\begin{array}{l}\text { José Luis } \\
\text { Esparza } \\
\text { Aguilar y } \\
\text { Domingo } \\
\text { García Pérez } \\
\text { de Lema. } \\
\end{array}$ & $\begin{array}{l}\text { La cultura de las } \\
\text { empresas familiares } \\
\text { turísticas mexicanas y } \\
\text { su influencia en la } \\
\text { gestión estratégica. }\end{array}$ \\
\hline 2. & $\begin{array}{l}\text { María de } \\
\text { Lourdes } \\
\text { Elena García, } \\
\text { Roberto } \\
\text { Hernández } \\
\text { Sampieri, } \\
\text { Benito } \\
\text { Erasmo } \\
\text { Vargas } \\
\text { Álvarez y } \\
\text { Héctor } \\
\text { Cuevas } \\
\text { Vargas. }\end{array}$ & $\begin{array}{l}\text { Diagnóstico de la } \\
\text { cultura organizacional } \\
\text { en universidades } \\
\text { tecnológicas bajo el } \\
\text { Modelo de Valores en } \\
\text { Competencia. }\end{array}$ \\
\hline 3. & $\begin{array}{l}\text { Tamara } \\
\text { Quiroz } \\
\text { Guzmán } \\
\text { David } \\
\text { Arnoldo } \\
\text { Valtiérrez }\end{array}$ & $\begin{array}{l}\text { Dimensiones } \\
\text { culturales en la } \\
\text { empresa estatal de } \\
\text { autoservicio, sucursal } \\
\text { Delicias, Chihuahua. }\end{array}$ \\
\hline
\end{tabular}




\begin{tabular}{|c|c|c|}
\hline & Ángel & \\
\hline 4. & $\begin{array}{l}\text { José Felipe } \\
\text { Ojeda } \\
\text { Hidalgo, } \\
\text { Sergio } \\
\text { Méndez } \\
\text { Valencia y } \\
\text { Roberto } \\
\text { Hernández } \\
\text { Sampieri. }\end{array}$ & $\begin{array}{l}\text { El liderazgo y su } \\
\text { relación con el } \\
\text { Modelo de Valores en } \\
\text { Competencia. }\end{array}$ \\
\hline 5. & $\begin{array}{l}\text { Tomás } \\
\text { Vargas } \\
\text { Halabí y } \\
\text { Marcela León } \\
\text { Madrigal. }\end{array}$ & $\begin{array}{ll}\text { Cultura nacional en } \\
\text { Costa Rica: Un } \\
\text { estudio exploratorio } \\
\text { descriptivo desde el } \\
\text { modelo de Hofstede. }\end{array}$ \\
\hline
\end{tabular}

Fuente: elaboración propia

El trabajo de (Esparza \& García, 2011), estudia la cultura de cuatro empresas familiares y se analiza su influencia en la gestión estratégica empleando el método propuesto por Cameron y Quinn (1999). Los resultados muestran que las empresas familiares con cultura de tipo ad hoc, de clan y jerárquico perciben valores similares, recurren a estrategias analizadoras y compiten en el mercado diferenciando sus productos y servicios; mientras que las empresas con cultura de mercado perciben el trabajo intensivo como valor primordial, recurren a estrategias defensivas y compiten especializándose en un segmento del mercado.

En el documento de (García, Hernández, Vargas, \& Cuevas, 2012), se estableció como objetivos principales de investigación: Validar el Modelo de los Valores en Competencia en dos universidades y establecer la cultura organizacional dominante y preferida en ambas instituciones. Resultó predominante la cultura actual de mercado en las dos instituciones, como cultura preferida la de clan en la universidad más reciente y adhocrática en la antigua.
El artículo de (Quiroz \& Valtiérrez, 2010), consistió en medir las dimensiones culturales de los grupos que confluyen en Delicias y la Región, bajo la perspectiva teórica de Hofstede, considerando cuatro dimensiones: eliminación de la incertidumbre, distancia en el poder, feminismo vs masculinidad, colectivismo vs individualismo, bajo la premisa de que una sociedad es de alto contexto cuando tiene una alta eliminación de la incertidumbre, alta distancia en el poder, es de valores altos en masculinidad y altamente colectiva.

(Ojeda, Méndez, \& Hernández, 2016), presentan los resultados de su investigación, los cuales corresponden a un análisis de 771 empresas de la región centro de México, en la cual se revisa la posible vinculación entre los cuadrantes del Modelo de Valores en Competencia (MVC) de Cameron y Quinn, y los estilos de liderazgo relacionados con estos cuadrantes.

Finalmente, (Vargas \& León, 2006), elaboran un estudio que retoma el modelo de cultura propuesto por Hofstede así como los resultados obtenidos para Costa Rica en su estudio original. Se comparan dichos valores con los resultados obtenidos en una muestra de 289 personas seleccionada por conveniencia entre estudiantes de maestría, asistentes a un seminario y empleados de una organización pública.

Tabla 3. Documentos teóricos revisados

\begin{tabular}{|l|l|l|}
\hline No. & \multicolumn{1}{|c|}{ Autores } & \multicolumn{1}{|c|}{ Título } \\
\hline 1. & $\begin{array}{l}\text { Paola } \\
\text { Podestá C. }\end{array}$ & $\begin{array}{l}\text { Un acercamiento al } \\
\text { concepto de } \\
\text { cultura. }\end{array}$ \\
\hline 2. & $\begin{array}{l}\text { Néstor Raúl } \\
\text { Porras } \\
\text { Velásquez }\end{array}$ & $\begin{array}{l}\text { Elementos básicos } \\
\text { para el análisis de } \\
\text { la cultura de las } \\
\text { organizaciones } \\
\text { desde la Psicología. }\end{array}$ \\
\hline 3. & $\begin{array}{l}\text { Armenio } \\
\text { Pérez }\end{array}$ & $\begin{array}{l}\text { Cultura } \\
\text { organizacional: }\end{array}$ \\
\hline
\end{tabular}




\begin{tabular}{|l|l|l|}
\hline & Martínez & $\begin{array}{l}\text { algunas reflexiones } \\
\text { a la luz de los } \\
\text { nuevos retos. }\end{array}$ \\
\hline 4. & $\begin{array}{l}\text { Rey Felipe } \\
\text { González } \\
\text { Meriño }\end{array}$ & $\begin{array}{l}\text { Cultura } \\
\text { organizacional y } \\
\text { valores Propuesta } \\
\text { para la medición }\end{array}$ \\
\hline 5. & $\begin{array}{l}\text { Penélope } \\
\text { Jaime } \\
\text { Santana y y y } \\
\text { Yasmina } \\
\text { Araujo } \\
\text { Cabrera }\end{array}$ & $\begin{array}{l}\text { Clima y cultura } \\
\text { organizacional: } \\
\text { iDos constructos } \\
\text { para explicar un } \\
\text { mismo fenómeno? }\end{array}$ \\
\hline
\end{tabular}

Fuente: elaboración propia

(Podestá, 2006), elabora un acercamiento al concepto de cultura desde dos perspectivas teóricas y propone una posibilidad de análisis a partir de las relaciones que se establecen entre conceptos relevantes de esas perspectivas.

Por su parte, (Porras, 2009) presenta una panorámica de los principales aspectos conceptuales y metodológicos en el abordaje de las culturas organizacionales. En la primera parte se hace alusión a los aspectos histórico-situacionales que llevaron a la idea de visualizar las organizaciones como "culturas". Luego discute la importancia del fenómeno cultural para la psicología de las organizaciones, en cuanto a su configuración, sus funciones y sus relaciones con otros fenómenos propios de la realidad psicosocial del trabajo.

En el trabajo orientado por (Pérez, 2009), se analiza el concepto de cultura organizacional a partir de la situación contemporánea de las organizaciones, así como describir la relación entre la cultura y la estrategia organizacional; para ello usa métodos teóricos histórico-lógico para el estudio de los antecedentes conceptuales.
(González R. , 2005), en este trabajo se expone un enfoque coherente de la relación entre los niveles de la cultura organizacional, para a partir del mismo conceptualizar y definir las producciones y/o conductas que permitan hacer mediciones de los valores dentro de una determinada organización.

Por último, (Jaime \& Araujo, 2007) en su trabajo sistematizan y clarifican la literatura existente sobre cultura y clima, para dilucidar la confusión terminológica y conceptual entre ambos constructos. Así mismo, a partir de dicha revisión abordan las principales diferencias y semejanzas entre ambos, para, finalmente presentar las principales limitaciones halladas tras la revisión de la literatura y las líneas de investigación futuras que podrían emprenderse para contribuir a superar dichas limitaciones.

\subsection{Modelos de medición de la cultura organizacional}

Es sabido por todos, que medir la cultura de la organización, ayuda a identificar las dimensiones de intervención organizacional, por ende, el medirla permite conocer el impacto que esta tiene sobre los resultados de la empresa, reconociendo que la cultura puede guiar los resultados de negocio de cualquier industria. Por consiguiente, debería ser una prelación para la alta dirección de cualquier organización, mostrar interés por contar con instrumentos cuantitativos o cualitativos que faciliten la medición de la cultura organizacional.

Aun cuando existen muchos modelos de medición de la cultura, algunos de ellos lo son: el modelo de orientación cultural de Hofstede, el modelo tridimensional de la cultura Payne, el modelo de Schein y el modelo de los valores en competencia 
(MVC) de Cameron y Quinn. De éstos, el que cuenta con mayor evidencia empírica (especialmente en América Latina) y posee mayor sencillez y claridad conceptual es, tal vez, el MVC (Hernández, Méndez, \& Contreras, 2014).

Sin embargo, medir y conocer la cultura organizacional de las empresas de hoy es algo complejo que no puede ser evidenciado sólo con la aplicación de un modelo o un cuestionario de los mencionados en el artículo, sino que se requiere de la incorporación de otras fuentes de información complementarias como observaciones, análisis de documentos, entrevista, entre otros (Tomás \& Rodríguez, 2009).

Lo anterior es consecuente con lo planteado por (García, Reyes, \& Hernández, 2005), quienes afirman la existencia de algunos métodos para la medición de la cultura organizacional, entre ellos se pueden mencionar: a) Enfoque basado en la observación, ya sea simple o compuesta; b) Determinando los valores, creencias, símbolos, rituales, historias y ceremonias; c) A través de la utilización de técnicas de muestreo no probabilístico como: accidental o por conveniencia, intencionado, caso típico, experto, entre otros; y d) La evaluación de la filosofía y estructura organizacional y del sistema de gestión organizacional (visión, misión, valores, políticas, objetivos, metas, acciones, etc.)

De otro lado (Vargas, 2007), en su investigación titulada "La culturocracia organizacional en México", presenta 15 métodos de medición de la cultura, de los cuales se muestran algunos en el Tabla 4.

Tabla 4. Métodos de medición

\begin{tabular}{|l|l|}
\hline Autores & $\begin{array}{l}\text { Métodos de medición de } \\
\text { la cultura }\end{array}$ \\
\hline Amsa (1986) & Métodos cuantitativos. \\
\hline
\end{tabular}

\begin{tabular}{|l|l|}
\hline $\begin{array}{l}\text { Barnett (1979, } \\
1988)\end{array}$ & $\begin{array}{l}\text { Método cuantitativo: } \\
\text { análisis de Galileo. }\end{array}$ \\
\hline $\begin{array}{l}\text { Bookblinder } \\
(1984)\end{array}$ & Método cuantitativo. \\
\hline Lewis (1992) & Combinación de métodos \\
\hline $\begin{array}{l}\text { Desatnick } \\
(1986)\end{array}$ & Método cuantitativo. \\
\hline $\begin{array}{l}\text { Hofstede } \\
(1986)\end{array}$ & Método cuantitativo \\
\hline $\begin{array}{l}\text { Reynierse } \\
(1986)\end{array}$ & Método cuantitativo \\
\hline $\begin{array}{l}\text { Tucker et al } \\
(1990)\end{array}$ & Cuestionario comprensivo \\
\hline Brink (1991) & $\begin{array}{l}\text { Teoría de la motivación } \\
\text { del código de color. }\end{array}$ \\
\hline
\end{tabular}

Fuente: elaboración propia a partir de (Vargas, 2007)

Llegado a este punto, se debe decir que, de entre todos los modelos utilizados para medir la cultura, en el artículo que aquí se presenta se hace una mención especial al modelo de valores en competencia. Tal modelo fue elegido por contar con abundante evidencia empírica y mayor claridad conceptual.

\subsubsection{Modelo de Valores en Competencia}

En principio, este modelo formulado por Kim S. Cameron y Robert E. Quinn, fue inicialmente desarrollado para especificar el criterio de efectividad organizacional, aunque posteriormente se utilizó para estudiar un amplio número de fenómenos corporativos como la cultura y el cambio, la comunicación, la ética y la administración de los sistemas de información, el liderazgo, la administración de programas de desarrollo de los recursos humanos, entre muchos otros (Ojeda, Méndez, \& Hernández, 2016).

Este modelo se basa en dos dimensiones que, en forma de ejes cartesianos, los cuales se trasponen produciendo cuatro cuadrantes 
cada uno con sus respectivos tipos de cultura, (ver figura 2).

\section{The Competing Values Framework}

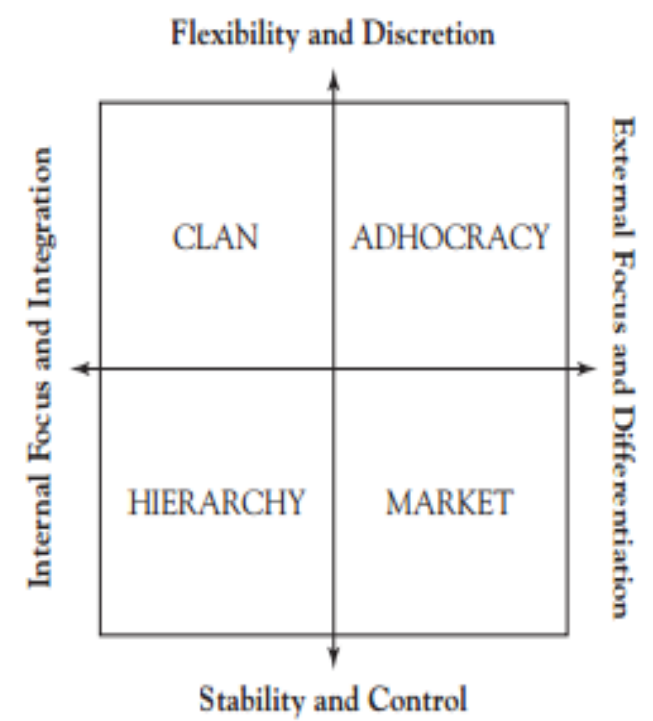

Figura 2. Modelo de Valores en Competencia

Fuente: (Cameron \& Quinn, 2006, pág. 35)

Cameron y Quinn presentan una descripción de los cuatro tipos culturales de la siguiente manera:

El tipo Clan, es muy similar al tipo de estructura familiar, es decir, que los miembros de la organización se perciben más bien como una familia extensa que como empresas que solo piensan en elevar los niveles de rentabilidad. Es propio del clan el trabajo en equipo ya que en este modelo organizacional se entiende que mientras más personas se alleguen de manera comprometida en la realización y el cumplimiento de una actividad, mejores y más efectivos serán los resultados para la empresa.

Según (Vivanco \& Franco , 2012), en este tipo clan la gerencia se puede considerar como ejemplo de buen tutor, consejero y padre de todos los que están dentro de la gran familia que es la empresa. A su vez, el estilo de dirección se caracteriza por promover el trabajo en equipo, el consenso y la participación. En definitiva, se genera un clima amigable cimentado en la lealtad.

El tipo Jerarquía, es propio de aquellas organizaciones cuya máximo interés se centra en la generación de eficiencia, confiabilidad y resultados predecibles. Los espacios de trabajo dentro de este modelo organizacional se caracterizan por ser estructurados y formalizados, donde se dejan ver la existencia de variados niveles jerárquicos en los prevalece la incorporación de procedimientos y normas como mecanismo de consulta permanente, por parte de todos los trabajadores, permitiéndoles un mayor desarrollo en la búsqueda del autocontrol.

El tipo Adhocracia (ausencia de la jerarquía), este modelo de organización surgió cuando el mundo dio el paso a la era informacional, según (Cameron \& Quinn, 2006) se refiere a un tipo ideal de organización, sensible a las turbulencias que caracterizan cada vez más el mundo del siglo XXI. En estas organizaciones se tiene como premisa que la iniciativa, la creatividad, la innovación, el espíritu empresarial y la preparación para el futuro deben ser la tarea de la alta dirección. Las organizaciones que se encasillan dentro de este tipo, tienen como característica su gran capacidad para reconfigurarse rápidamente cuando surgen nuevas circunstancias, es decir, que en su interior se fomenta la adaptabilidad, la flexibilidad y la creatividad como herramientas para enfrentar la incertidumbre, la ambigüedad y la sobrecarga de información típicas de la modernidad.

El tipo Mercado, esta organización se enfoca hacia el ambiente externo, generando 
vínculos con las entidades del entorno como clientes y consumidores, proveedores, sindicatos, organismos reguladores y competidores. En palabras de Cameron y Quinn, el foco principal de este tipo de empresas es realizar transacciones (Intercambios, ventas, contratos) con otros grupos, para de esta manera crear ventaja competitiva; por esta razón los valores que dominan las organizaciones de mercado son la competitividad y la productividad.

En resumidas cuentas, en el trabajo de (Cameron \& Quinn, 2006), se descubrieron que los cuatro cuadrantes que surgieron de estos análisis coinciden exactamente con las principales formas organizativas en la ciencia organizacional y a su vez coinciden con teorías clave de gestión sobre el éxito de la organización, los enfoques de la calidad organizacional, los roles de liderazgo y las habilidades gerenciales.

La figura 3 muestra las características más distintivas de cada de cultura, haciendo énfasis en: el tipo de cultura, la orientación, el tipo de líder, los valores y la teoría de efectividad.

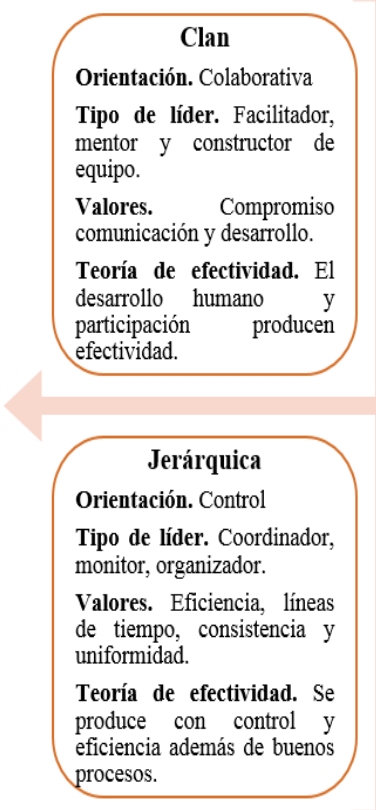

Figura 3. Caracterización de cada tipo de cultura Fuente: elaboración propia a partir de (Cameron \& Quinn, 2006, pág. 46)

\section{Discusión y conclusión}

Desde los antecesores lejanos de la corriente de la cultura organizacional, se han propuesto distintas definiciones a lo largo de los años, pero en todas ellas hay más elementos en común que en discordia, y con los cuáles se pudo generar una nueva definición como la que sigue: "intangible del que hacen parte las creencias, los valores, las costumbres y rituales que reflejan la mentalidad que predomina en la organización y que a su vez le garantiza la heterogeneidad".

Se deja ver en la revisión bibliográfica efectuada, que no existe un único modelo o enfoque para medir la cultura organizacional, a lo cual se suma según Hernández, Fernández, y Baptista citado por (García, Reyes, \& Hernández, 2005) que ningún modelo es completamente incluyente, ni totalmente correcto, pero aquél que se seleccione debe describir la realidad con exactitud, ser válido y además que pueda integrar y organizar la mayoría de las dimensiones propuestas. Lo importante es que el modelo que decida usar la organización para medir su cultura logre incorporar la mayoría de dimensiones posibles tanto desde la perspectiva interna como externa.

Llegado a este punto, es clave señalar que este manuscrito no debe terminar simplemente con una revisión bibliográfica de los modelos usados para medir la cultura organizacional, sino que es necesario llegar hasta el establecimiento de verdaderas acciones que permitan mejorar el 
desempeño organizacional de las empresas, permitiéndoles de esta manera prepararse adecuadamente para enfrentar las circunstancias del entorno.

Es evidente que no es lo mismo medir la cultura organizacional de una empresa colombiana que de una empresa estadounidense, peruana, venezolana o ecuatoriana, ya que sus valores, costumbres, necesidades, creencias, expectativas y un largo, etc. son diferentes. Sin embargo, no se debe ceder en el convencimiento de que se requiere medir la cultura organizacional para mejorar la eficiencia, eficacia y efectividad de los procesos organizacionales, independiente de que ésta pueda variar por niveles (industrias nacionales y regionales), tipos, jerarquías e incluso por género, profesión, aspectos étnicos y religiosos.

Con base en la posición de diferentes autores, es posible afirmar que no existen modelos, métodos e instrumentos que permitan valorar y medir con precisión la cultura organizacional de cualquier tipo de empresa, en razón a los permanentes cambios en el comportamiento de las personas y del contexto, sin embargo es apremiante que las organizaciones se apropien de cualquier modelo o instrumento de medición de carácter cualitativo o cuantitativo para evaluar su cultura organizacional.

En suma, se concluye que de acuerdo a la revisión bibliográfica efectuada para este escrito, en el caso colombiano se requiere de una mayor cantidad de estudios sobre la medición de cultura organizacional, que conduzcan a la identificación de nuevas formas de medirla, ya que, en los últimos años Colombia experimentará una serie de cambios económicos, sociales, ambientales, tecnológicos y políticos seguramente producidos por el postconflicto.

\section{BIBLIOGRAFÍA}

Aktouf, O. (2002). El Simbolismo y la "Cultura Organizacional" De los abusos conceptuales a las lecciones de campo. Administer(1), 63-93.

Cambridge University Press. (febrero de 2017). Obtenido de http://dictionary.cambridge.org/es/di ccionario/ingles/ultimate

Cameron, K., \& Quinn, R. (2006). Diagnosing and Changing Organizational Culture. JosseyBass.

Esparza, J., \& García, D. (2011). La cultura de las empresas familiares turísticas mexicanas y su influencia en la gestión estratégica. Cuadernos de Administración, 295-313.

García, L., Hernández, R., Vargas, E., \& Cuevas, H. (2012). Diagnóstico de la cultura organizacional en universidades tecnológicas bajo el Modelo de Valores en Competencia. Estudios en Ciencias Sociales y Administrativas de la Universidad de Celaya, 2, 9-29.

García, L., Reyes, G., \& Hernández, T. (2005). Validación de un instrumento de medición que analiza la cultura organizacional desde dos perspectivas. RECAI Revista de Estudios en Contaduría, 
Administración e Informática., 2142.

Góngora , N., Nóbile, C., \& Reija, L. (2014). Estudio comparativo de la cultura organizacional. Ciencias Administrativas FCE UNLP(4).

González, R. (2005). Cultura organizacional y valores. Propuesta para la medición. Folletos Gerenciales, 2741.

Hernández, R., \& Méndez, S. (2012). Exploración factorial del clima y la cultura organizacional. PsiqueMag, $1-37$.

Hernández, R., Méndez, S., \& Contreras, R. (2014). Construcción de un instrumento para medir el clima organizacional en función del modelo de los valores en competencia. Contaduría y Administración, 59(1), 229-257.

Jaime , P., \& Araujo, Y. (2007). Clima y cultura organizacional: ¿Dos constructos para explicar un mismo fenómeno? Palma, España: Decisiones basadas en el conocimiento y en el papel social de la empresa.

Ojeda, J., Méndez, S., \& Hernández, R. (2016). El liderazgo y su relación con el Modelo de Valores en Competencia. TELOS. Revista de Estudios Interdisciplinarios en Ciencias Sociales, 17-38.

Pérez, A. (2009). Cultura organizacional: algunas reflexiones a la luz de los nuevos retos. Revista Venezolana de Gerencia, 183-194.

Peters, T., \& Waterman, R. (1984). En búsqueda de la excelencia. Bogotá: Norma.

Podestá, P. (2006). Un acercamiento al concepto de cultura. Cuad. Difus., 25-39.

Porras, N. (2009). Elementos básicos para el análisis de la cultura de las organizaciones desde la Psicología. Tesis Psicológica, 36 - 51.

Quiroz, T., \& Valtiérrez, D. (2010). Dimensiones culturales en la empresa estatal de autoservicio, sucursal Delicias, Chihuahua. Revista Mexicana de Agronegocios, 375-386.

Real Academia Española . (2001). Diccionario de la lengua española. Madrid: Espasa.

Rodríguez, D. (2001). Gestión Organizacional. Elementos para su estudio. Santiago: Universidad Católica de Chile.

Romero, J., Matamoros, S., \& Campo, C. (2013). Sobre el cambio organizacional. Una revisión bibliográfica. Innovar, 35-52.

Ruiz, Y., \& Naranjo, J. (2012). La investigación sobre cultura organizacional en Colombia: una mirada desde la difusión en revistas científicas. Diversitas: Perspectivas 
en Psicología, vol. 8, núm. 2, 2012, pp. 285-307, 285-307.

Schein, E. (2009). The Corpotare Culture Survival Guide. San Francisco: Jossey-Bass.

Serna, H. (2003). Gerencia Estratégica. Bogotá.

Stephen, R. (2004). Comportamiento Organizacional. México: Pearson.

Toca, C., \& Carrillo, J. (2009). Asuntos teóricos y metodológicos de la cultura organizacional. Civilizar, 9(17), 117-136.

Tomás, M., \& Rodríguez, D. (2009). Conocer la cultura de la universidad contemporánea: el CICOU. Revista Iberoamericana de Educación, 1-12.

Universidad de la Experiencia de Zaragoza. (2017). UEZ. Obtenido de https://uez.unizar.es/sites/uez.unizar. es/files/users/pjulian/TrabajosTaller/ PowerPoints/introduccion_a_la_etim ologia_grecolatina.pdf

Vargas, J. (2007). La culturocracia organizacional en México. México: Eumed. net.

Vargas, T., \& León, M. (2006). Cultura nacional en Costa Rica: Un estudio exploratorio descriptivo desde el modelo de Hofstede. Revista Costarricense de Psicología, 25(38), 55-60.

Vivanco, J., \& Franco , R. (Octubre de 2012). Los tipos de cultura organizacional y el rendimiento de las pymes en Aguascalientes. XVII Congreso Internacional de Contaduría, Administración e Informática. 